\title{
Association between MYH9 and APOL1 Gene Polymorphisms and the Risk of Diabetic Kidney Disease in Patients with Type 2 Diabetes in a Chinese Han Population
}

\author{
Hailing Zhao $\mathbb{D}^{1},{ }^{1}$ Liang Ma $\mathbb{D}^{2}{ }^{2}$ Meihua Yan $\mathbb{D}^{1},{ }^{1}$ Yan Wang, ${ }^{1,3}$ Tingting Zhao $\mathbb{D}^{1}{ }^{1}$ \\ Haojun Zhang $\mathbb{D}^{1},{ }^{1}$ Peng Liu, ${ }^{1,3}$ Yanzhen Liu, ${ }^{1}$ and Ping Li ${ }^{1}{ }^{1}$ \\ ${ }^{1}$ Beijing Key Lab for Immune-Mediated Inflammatory Diseases, Institute of Clinical Medical Science, China-Japan Friendship \\ Hospital, Beijing, China \\ ${ }^{2}$ Clinical Laboratory, China-Japan Friendship Hospital, Beijing, China \\ ${ }^{3}$ Graduate School of Peking Union Medical College, Chinese Academy of Medical Science and Peking Union Medical College, \\ Beijing, China
}

Correspondence should be addressed to Ping Li; 1p8675@163.com

Received 21 December 2017; Accepted 1 April 2018; Published 10 May 2018

Academic Editor: Secundino Cigarran

Copyright (C) 2018 Hailing Zhao et al. This is an open access article distributed under the Creative Commons Attribution License, which permits unrestricted use, distribution, and reproduction in any medium, provided the original work is properly cited.

\begin{abstract}
Single-nucleotide polymorphisms (SNPs) in MYH9-APOL1 gene regions have been reported to be associated with diabetic kidney disease (DKD) in the American population. We examined the association between polymorphisms in MYH9-APOL1 and DKD susceptibility in a Chinese Han population. MYH9 rs3752462 (T>C) and APOL1 rs136161 (C>G) were genotyped in 303 DKD patients and 364 type 2 diabetes mellitus (T2DM) patients without kidney disease using the TaqMan SNP genotyping assay. Chi-squared test and multivariate logistic regression were used to evaluate the association. We observed that only MYH9 rs3752462 was associated with DKD (genotype, $P=0.004$; allele, $P=0.002$ ). Genetic model analysis revealed that rs 3752462 was associated with increased risk of DKD under a dominant model adjusted by age and sex (adjusted odds ratio (aOR), 1.675; 95\% CI 1.225-2.289; $P=0.001$ ) and an additive model (TC versus TT: aOR, 1.649; 95\% CI 1.187-2.290; CC versus TT: aOR, 1.817; 95\% CI 0.980-3.367; $P=0.005)$. The combined effect of rs3752462 TC + rs136161 CC genotype showed an association of DKD adjusted by age and sex $(\mathrm{aOR}, 1.732$; $95 \%$ CI 1.128-2.660; $P=0.012)$. After a Holm-Bonferroni correction for multiple tests, the $\mathrm{C}$ allele frequencies of the rs 3752462 and the TC + CC genotype in the dominant model were considered statistically significant with a markedly increased risk of DKD $(P<0.00208 ; P<0.002)$. Our results suggest that MYH9 rs 3752462 is significantly associated with an increased risk of DKD in Chinese Han individuals.
\end{abstract}

\section{Introduction}

Diabetic kidney disease (DKD) is a common and serious microvascular complication of diabetes mellitus (DM), which is characterized by an elevated urinary albumin excretion rate, elevated blood pressure, and declined renal function. Approximately $30-40 \%$ of DM patients will develop $\mathrm{DKD}$, which is the leading cause of end-stage renal disease (ESRD) and renal failure [1]. Genetic factors appear critical in its pathogenesis based upon the evidence including aggregation in families, variable incidence rates of DKD between different races, and the highly heritable nature of diabetic renal clinic and histologic changes [2]. Compared to the Caucasian population, the Asia populations are more likely to suffer from DKD [3]. Identification of potentially susceptible genes and loci is needed to facilitate earlier identification and prevention of $\mathrm{DKD}$, particularly in China.

The MYH9 gene located on chromosome 22 q12.313.2 encodes nonmuscle myosin IIA. The approximately $224 \mathrm{kDa}$ protein is widely expressed in most cells in the body [4, 5]. Polymorphisms in MYH9 have been strongly associated with ESRD according to genome-wide association studies (GWAS), including human immunodeficiency 
virus- (HIV-) associated nephropathy in African Americans and idiopathic focal segmental glomerulosclerosis in European Americans and Hispanic Americans. Multiple common single-nucleotide polymorphisms (SNPs) in MYH9 are associated with a greater risk for nondiabetic ESRD [6-8]. The APOL1 gene, which is also located on chromosome 22, encodes apolipoprotein L-1. This gene has been associated with kidney disease in African Americans [9]. APOL1 gene polymorphisms have also been more intensely associated with the risk of kidney disease previously attributed to MYH9 [10]. The two genes cosegregate in many populations, which makes it difficult to differentiate between the two association signals.

A few studies reported the association of MYH9 or APOL1 with DKD. A GWAS and transethnic meta-analysis established the significant associations of $\mathrm{MYH9}$ and APOL1 on chromosome 22 q12.3 with DKD in European American, African American, and American Indian populations. Furthermore, MYH9 (rs5750250)-APOL1 (rs136161) contributed the strongest association with DKD in African American populations [11]. Another study reported the association of four MYH9 SNPs (rs4821480, rs2032487, rs4281481, and rs3752462) with T2DM-ESRD susceptibility in European Americans [8]. However, among them, only MYH9 rs3752462 and APOL1 rs136161 present genetic polymorphisms in Chinese Han individuals.

The susceptibility of MYH9 and APOL1 polymorphisms with DKD in Chinese populations has not been well studied. Considering the strong ethnic heterogeneity for gene polymorphisms, we evaluated the association of MYH9 rs3752462 (T>C) and APOL1 rs136161 (C>G) with DKD in a Chinese Han population.

\section{Materials and Methods}

2.1. Subjects. The clinic-based, case-control study recruited a total of 667 volunteers with T2DM. Among them, 303 patients with a history of DKD were defined as the case group. The remaining 364 participants, who had been diagnosed as T2DM for at least 7 years and had no history of $\mathrm{DKD}$, were defined as the control group, regardless of age and sex.

Type 2 diabetes patients were diagnosed according to the 2012 American Diabetes Association diagnostic criteria; DKD patients were defined by the National Kidney Foundation Kidney Disease Outcomes Quality Initiative (NKF K-DOQI) guidelines.

This study was approved by the institutional ethics committee of the China-Japan Friendship Hospital (Beijing, China), and written informed consent was obtained from all individuals.

2.2. DNA Isolation and Genotyping. Genomic DNA was extracted from peripheral blood using the QIAamp DNA Blood Mini Kit (Qiagen, Hilden, Germany) in accordance with the manufacturer's protocol and quantified using a NanoDrop 1000 spectrophotometer (Thermo Scientific, Waltham, MA, USA). Genotyping was confirmed using the TaqMan SNP genotyping assay (Applied Biosystems,
Waltham, MA, USA) and the ABI PRISM 7500 Sequence Detection System (Applied Biosystems).

Polymerase chain reaction (PCR) amplification was performed in a $25 \mu \mathrm{L}$ reaction mixture containing $50 \mathrm{ng}$ DNA, $12.5 \mu \mathrm{L}$ of Premix Ex Taq (Takara, Shiga, Japan), 5 pmol of each primer (Applied Biosystems), and $3 \mathrm{pmol}$ of each probe (Applied Biosystems). The amplification conditions consisted of 40 cycles of $95^{\circ} \mathrm{C}$ for $10 \mathrm{~min}, 92^{\circ} \mathrm{C}$ for 15 seconds, and $60^{\circ} \mathrm{C}$ for $1 \mathrm{~min}$. The primers used to detect SNPs were synthesized by Applied Biosystems.

To verify genotypes, the PCR products were randomly selected for DNA sequencing analysis by TsingKe Biological Technology (Beijing, China) and the results were compared with the results of TaqMan genotyping. The primers used for the PCR were rs3752462, 5'-AAGACACCTCCACAAC CAACAC- $3^{\prime}$ (forward) and $5^{\prime}$-GCTCTTCAACCACACCAT GTTC-3' (reverse), and rs136161, 5' -CTCTCTTGCTG GCT TATGGAA-3' (forward) and $5^{\prime}$-GCTGTGATGTGGGACT TGTTT-3' (reverse).

2.3. Statistical Analyses. Clinical data, including age, gender, body mass index (BMI), blood pressure, duration of diabetes, A1C, total cholesterol (TC), high-density lipoprotein cholesterol (HDL-C), low-density lipoprotein cholesterol (LDL-C), triglyceride (TG), and homocysteine (Hcy), were non-Gaussian distributed. Wilcoxon signed-rank test was used to analyze the differences in clinical characteristics of the DKD and diabetic groups, and the data are presented as median (interquartile range). The Hardy-Weinberg equilibrium analysis of both SNPs was conducted using the Chi-squared test. The genotype and allelic frequencies of SNPs were also assessed by the Chi-squared test.

Multivariate logistic regression was carried out to analyze the association between each SNP and susceptibility to DKD after adjustment for age and gender in the additive, recessive, or dominant models. Multivariate logistic regression was also used for the combined effect of both MYH9 rs3752462 and APOL1 rs136161 polymorphism on DKD. An example to define these genetic models is the rs3752462 SNP, where C is the minor allele. For the dominant model, CC and TC were coded as 1 and TT was coded as 0. For the recessive model, CC was coded as 1 and TC and TT were coded as 0 . For the additive model, CC, TC, and TT were coded as 2, 1, and 0 , respectively. For SNP rs136161, G is the minor allele.

The Holm-Bonferroni correction was started by ordering the $P$ values (from lowest to highest) as $P_{(1)} \ldots P_{(m)}$ and let the associated hypotheses be $H_{(1)} \ldots H_{(m)}$. For a given significance level $\alpha$, let $\kappa$ be the minimal index such that $P>\alpha$ / $(m+1-\kappa)$. Reject the null hypotheses $H_{(1)} \ldots H_{(\kappa-1)}$ and reject $H_{(\kappa)} \ldots H_{(m)}$. In present research, association analysis was performed for 26 times, $m=26$.

\section{Results}

3.1. Subjects. A total of 667 T2DM participants were included, including 303 patients with a history of DKD and 364 patients without a history of kidney disease. The clinical characteristics of the participants are listed in Table 1. 
TABle 1: Demographics and clinical characteristics of T2DM patients with and without kidney diseases.

\begin{tabular}{lccc}
\hline Variables & DKD $(n=303)^{\mathrm{a}}$ & DM $(n=364)^{\mathrm{a}}$ & $P$ \\
\hline Age $(\mathrm{y})$ & $63.0(55.0,72.0)$ & $61.0(54.0,68.0)$ & 0.002 \\
Sex, male $(\%)$ & $63.4(192 / 303)$ & $58.0(211 / 364)$ & 0.156 \\
BMI $\left(\mathrm{kg} / \mathrm{m}^{2}\right)$ & $25.82(24.0,28.23)$ & $25.29(23.2,27.78)$ & 0.008 \\
Duration of diabetes $(\mathrm{y})$ & $15.0(9.0,21.0)$ & $13.0(10.0,18.0)$ & 0.039 \\
History of hypertension $(\%)$ & $77.56(235 / 303)$ & $48.08(175 / 364)$ & $<0.001$ \\
Current smoking $(\%)$ & $33.0(100 / 303)$ & $26.6(97 / 364)$ & 0.073 \\
SBP $(\mathrm{mmHg})$ & $138.0(125.0,150.0)$ & $126.0(120.0,139.8)$ & $<0.001$ \\
DBP $(\mathrm{mmHg})$ & $80.0(75.0,84.0)$ & $7.0(70.0,80.0)$ & 0.026 \\
A1C $(\%)$ & $8.0(6.7,9.35)$ & $11.49(9.54,9.2)$ & 0.037 \\
Hcy $(\mu \mathrm{mol} / \mathrm{L})$ & $13.89(11.09,17.22)$ & $4.17(3.55,4.92)$ & $<0.001$ \\
TC $(\mathrm{mmol} / \mathrm{L})$ & $4.21(3.49,5.06)$ & $1.02(0.85,1.24)$ & 0.51 \\
HDL-C $(\mathrm{mmol} / \mathrm{L})$ & $0.96(0.78,1.18)$ & $2.38(1.91,2.96)$ & 0.004 \\
LDL-C $(\mathrm{mmol} / \mathrm{L})$ & $2.34(1.86,3.05)$ & $1.43(1.0,2.17)$ & 0.74 \\
TG $(\mathrm{mmol} / \mathrm{L})$ & $1.74(1.22,2.57)$ & $<0.001$ \\
\hline
\end{tabular}

Abbreviations: A1C, hemoglobin A1C; BMI, body mass index; DBP, diastolic blood pressure; Hcy, homocysteine; HDL-C, high-density lipoprotein cholesterol; LDL-C, low-density lipoprotein cholesterol; SBP, systolic blood pressure; TC, total cholesterol; TG, triglyceride. ${ }^{\mathrm{a}}$ Data are shown as median (interquantile range) or \%. $P<0.05$ indicates statistical significance.

There were no significant differences in gender, smoking, TC, and LDL-C between the two groups. However, there were significant differences in age, BMI, duration of diabetes, blood pressure, A1C, Hcy, HDL-C, and TG between the DKD and T2DM groups.

3.2. Genotype and Allele Distributions of MYH9 rs3752462 and APOL1 rs136161 Polymorphisms. The genotype and allele frequencies of the rs3752462 and rs136161 polymorphisms in DKD and T2DM groups are shown in Table 2. Both SNPs were in Hardy-Weinberg equilibrium $(P>0.05)$, and their minor allele frequencies were $>5 \%$. The genotype and allele frequencies of the rs3752462 were different between DKD and T2DM groups (genotype, $P=0.004$; allele, $P=0.002)$. A significant association with the increased risk of DKD remained for the minor allele $C$ after a HolmBonferroni correction $(P<0.00208)$. However, there was no significant differences in the genotype and allele frequencies of the rs136161 between the two groups (genotype, $P=0.944$; allele, $P=0.751)$.

3.3. Association of MYH9 rs3752462 and APOL1 rs136161 Polymorphisms with DKD. Different genetic models were applied to verify the associations of MYH9 rs3752462 and APOL1 rs136161 polymorphisms with DKD (Table 3). We assumed that the minor alleles of both SNPs were the risk factors compared to the common alleles. In the dominant model, multivariate logistic regression analysis revealed that when the rs3752462 TT genotype was used as the reference, the TC+CC genotype was associated with a high risk of DKD (TC + CC versus TT: odds ratio (OR), 1.690; 95\% CI 1.240-2.303; $P=0.001)$. In the additive model, when the rs3752462 TT homozygote genotype was used as the reference, the TC and CC genotypes were associated with a decreased risk of DKD (TC versus TT: OR, 1.684; 95\% CI 1.216-2.332; CC versus TT: OR, 1.720; 95\% CI 0.935-3.162;
$P=0.004)$. In the recessive model, when the rs3752462 TT + TC genotype was used as the reference, the CC genotype was not associated with the risk of DKD (CC versus TT + TC: OR, 1.398; 95\% CI 0.772-2.533; $P=0.269$ ). The results were remained similar after adjusted by age and sex. After the Holm-Bonferroni correction, only the TC + CC genotype showed significant association with an increased risk of DKD $(P<0.002)$. There was no significant association between APOL1 rs136161 and the risk of DKD under these genetic models, which was remained similar after adjustment for age and sex (Table 3).

3.4. Combined Effect of MYH9 rs3752462 and APOL1 rs136161 Polymorphisms on DKD. Multivariate logistic regression analysis was applied to analyze the combined effect of both SNPs on DKD (Table 4). When the rs3752462 TT + rs136161 CC genotype was used as the reference, we found that the patients with rs3752462 TC + rs136161 CC genotype showed a higher risk of DKD (OR, 1.734; 95\% CI 1.134-2.652; $P=0.011$ ). The combined effect of other genotypes of rs3752462 and rs136161 was also examined; however, no difference was found. After adjustment by age and sex, the results were similar (Table 4). However, there was no significant association after Holm-Bonferroni correction.

\section{Discussion}

DKD is one of the most frequent microvascular complications of diabetes and is the leading cause of ESRD worldwide. Genetic heterogeneity and gene-gene or gene-environment interactions are frequently hypothesized as being important in this complex genetic disorder [12]. In the present study, 667 participants (303 DKD patients and 364 DM patients) were enrolled to investigate associations of $M Y H 9$ rs3752462 and APOL1 rs136161 with the risk of DKD in a 
TABLE 2: Genotype and allele frequency of SNPs rs3752462 and rs136161 between DKD patients $(n=303)$ and DM controls $(n=364)$.

\begin{tabular}{|c|c|c|c|c|c|c|c|c|}
\hline \multirow[b]{2}{*}{ rs3752462 } & \multicolumn{5}{|c|}{ Genotypes, $n(\%)$} & \multicolumn{3}{|c|}{ Alleles, $n(\%)$} \\
\hline & TT & TC & $\mathrm{CC}$ & HWE $P$ value & $P$ & $\mathrm{~T}$ & $\mathrm{C}$ & $P$ \\
\hline $\mathrm{DM}$ & $227(62.4 \%)$ & $115(31.6 \%)$ & $22(6.0 \%)$ & 0.155 & 0.00 & $569(57.1 \%)$ & 159 (42.9\%) & \\
\hline DKD & $150(49.5 \%)$ & $128(42.2 \%)$ & $25(8.3 \%)$ & 0.752 & & $428(70.6 \%)$ & $178(29.4 \%)$ & 0.002 \\
\hline rs136161 & $\mathrm{CC}$ & CG & GG & HWE $P$ value & $P$ & $\mathrm{C}$ & G & $P$ \\
\hline $\mathrm{DM}$ & $213(58.5 \%)$ & $126(34.6 \%)$ & $25(6.9 \%)$ & 0.287 & & $552(75.8 \%)$ & $176(24.2 \%)$ & \\
\hline DKD & $180(59.4 \%)$ & $104(34.3 \%)$ & $19(6.3 \%)$ & 0.449 & 0.944 & $464(76.6 \%)$ & $142(23.4 \%)$ & 0.151 \\
\hline
\end{tabular}

${ }^{*} P<0.05 ; \Delta$ indicates statistical significance by Holm-Bonferroni correction; HWE: Hardy-Weinberg equilibrium.

TABLE 3: Genetic model analyses of the association between the SNPs and DKD with adjustment for age and gender.

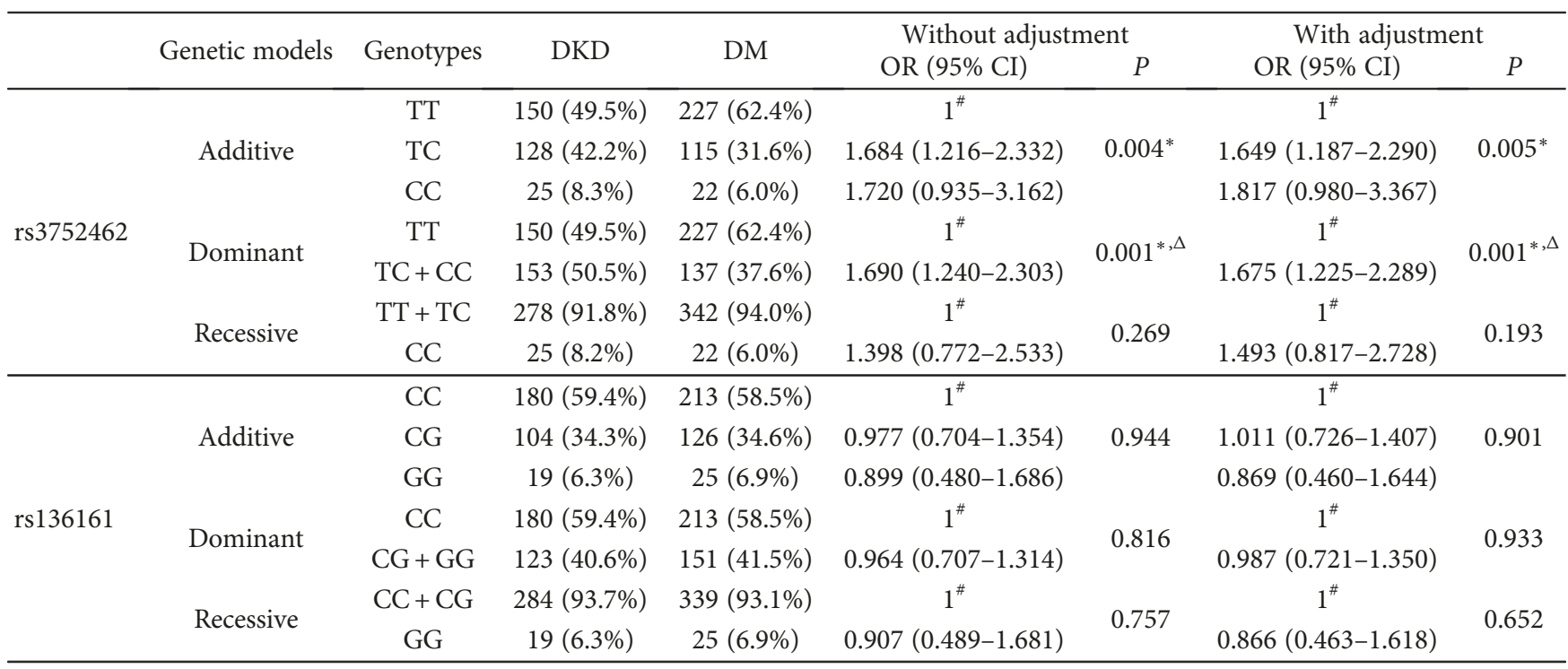

Abbreviations: ORs, odds ratios; CI, confidence interval. ${ }^{*}$ Reference category (odds ratio, 1.0 ); ${ }^{*} P$ value $<0.05 ; \Delta$ indicates statistical significance by HolmBonferroni correction.

TABLE 4: The combined effect of MYH9 rs3752462 and APOL1 rs136161 polymorphisms on DKD.

\begin{tabular}{|c|c|c|c|c|c|c|c|}
\hline \multicolumn{2}{|c|}{ Genotypes } & \multirow{2}{*}{$\begin{array}{c}\text { DKD } \\
303\end{array}$} & \multirow{2}{*}{$\begin{array}{l}\text { DM } \\
364\end{array}$} & \multicolumn{2}{|c|}{ Without adjustment } & \multicolumn{2}{|c|}{ With adjustment } \\
\hline rs3752462 & rs136161 & & & OR $(95 \% \mathrm{CI})$ & $P$ & OR $(95 \% \mathrm{CI})$ & $P$ \\
\hline TT & $\mathrm{CC}$ & 89 & 134 & $1^{\#}$ & - & $1^{\#}$ & - \\
\hline $\mathrm{TT}$ & $\mathrm{CG}+\mathrm{GG}$ & 61 & 93 & $0.988(0.649-1.503)$ & 0.953 & $1.039(0.679-1.588)$ & 0.861 \\
\hline TC & $\mathrm{CC}$ & 76 & 66 & $1.734(1.134-2.652)$ & $0.011^{*}$ & $1.732(1.128-2.660)$ & $0.012^{*}$ \\
\hline TC & $\mathrm{CG}+\mathrm{GG}$ & 52 & 49 & $1.598(0.995-2.565)$ & 0.052 & $1.596(0.989-2.575)$ & 0.055 \\
\hline $\mathrm{CC}$ & $\mathrm{CC}$ & 15 & 13 & $1.737(0.789-3.826)$ & 0.170 & $1.923(0.864-4.283)$ & 0.109 \\
\hline $\mathrm{CC}$ & $\mathrm{CG}+\mathrm{GG}$ & 10 & 9 & $1.673(0.654-4.281)$ & 0.283 & $1.736(0.669-4.503)$ & 0.257 \\
\hline
\end{tabular}

${ }^{\#}$ Reference category (odds ratio, 1.0); ${ }^{9}$ Adjustment for age and gender; ${ }^{*} P$ value $<0.05$.

Chinese Han population. Our results indicate that the minor allele of SNP rs3752462 is associated with an increased risk of DKD, while APOL1 rs136161 was not significantly associated with DKD. The results suggest that MYH9 rs3752462 might play an important role in the risk of DKD in the Chinese Han population.

The first association study of MYH9 with kidney disease was observed in the patients with the giant platelet syndromes, a group of diseases caused by MYH9 mutations and with a spectrum of abnormalities including low platelet count, hearing loss, giant platelets, and cataract may present focal segmental glomerular sclerosis (FSGS) [7]. APOL1 is a neighbor gene presenting very strong cosegregation with MYH9 in African descendants. Two studies reported stronger association with CKD of APOL1 than $M Y H 9$, being the marker possibly responsible for the effect previously attributed to MYH9 [9, 13]. Previous studies suggested that common polymorphisms in MYH9 were strongly associated 
with nondiabetic kidney diseases in several ethnic populations [14-16]. However, polymorphisms in MYH9 have been proven to be associated with diabetic nephropathy in 1963 European Americans, including 536 cases with T2DM-ESRD and 1427 nonnephropathy controls [8]. A recent GWAS and transethnic meta-analysis showed that SNPs in the MYH9-APOL1 gene region showed the strongest association with DKD in African Americans, which provided suggestive evidence for association with DKD, although it did not reach genome-wide significance $\left(P<5 \times 10^{-8}\right)$ [11]. Our results indicated that $M Y H 9$ rs3752462 was strongly associated with clinically diagnosed DKD in a Chinese Han population, and the minor allele $\mathrm{C}$ contributed to the increased risk of $\mathrm{DKD}$, which might be due to genetic heterogeneity. Although the genotype and allele frequencies of APOL1 rs136161 were not associated with DKD, SNP rs136161 CC genotype combined with SNP rs3752462 TC genotype showed association with the risk of $\mathrm{DKD}$, providing further evidence that single genetic abnormalities were rarely the only cause of complex disease.

MYH9 encodes the nonmuscle myosin heavy chain 9, which forms myosin II with other subunits. Myosin II, a motor protein, binds actin to regulate cellular motility. MYH9 is mainly expressed in the podocytes, as well as in mesangial cells and arteriolar and peritubular capillaries in kidneys. As a motor protein, abnormal MYH9 expression, localization, or function change will lead to cytoskeleton damage, further causing proteinuria or renal failure in patients with CKD [17-19]. Classical deletion of Myh9 in mice results in embryonic lethality due to the loss of cell-cell adhesion and cell movement during gastrulation [20, 21]. Podocytespecific deletion of Myh9 in C57BL/6 mice causes significant susceptibility to experimental doxorubicin hydrochloride glomerulopathy [22]. A common missense mutation in MYH9 (E1841K) alters podocyte cytoskeletal structure and renders podocytes more susceptible to injury after a damaging stimulus [23]. However, the mechanisms responsible for them have not been defined. Wasik et al. found that the activity of myosin II was reduced by septin 7, which could hinder GLUT4 storage vesicle and fusion with the plasma membrane, reduce glucose uptake into podocytes, and further cause insulin resistance [24]. Fan et al. showed that NM-IIA activity was also inhibited by SLIT2/ROBO2 signaling, which could reduce podocyte adhesion in kidney glomeruli and aggravate the injuries of the glomerular filtration barrier in patients with CKD [25].

SNP rs3752462 is located in intron 13 of MYH9, and the functional effect has not been reported. One possibility was that the mutation in noncoding areas modulated gene transcription or pre-mRNA splicing. Furthermore, MYH9 rs3752462 might directly regulate gene expression or on a more distant gene (such as APOL1). It has been reported that the strongest kidney disease association was mapped to the region of introns $13-15$ by genotyping 79 MYH9 SNPs in a total of 2496 cases (FSGS, HIV-associated nephropathy, and ESRD attributed to hypertension) and healthy controls [26]. Therefore, SNP rs3752462 in MYH9 might be a functional variation or just a tag SNP in strong linkage disequilibrium with the causal functional SNP. This hypothesis needs confirmation in a future study.

Recently, MYH9 rs3752462 was associated with cerebrovascular blood flow $(\mathrm{CBF})$ in patients with type 2 diabetes [27]. Both cerebrovascular disease and DKD were the main vascular complications of T2DM, which implies similar molecular mechanisms in the association of MYH9 rs3752462 with cerebrovascular disease and DKD. In addition, the $\mathrm{T}$ allele of SNP rs3752462 was associated with an excess risk for high blood pressure in patients with CKD in a Chinese population. It was also revealed that SNP rs3752462 was an independent predictor of a reduced glomerular filtration rate in the Spanish RENASTUR cohort population [28]. Therefore, blood pressure or glomerular filtration rate might be involved in the risk of MYH9 rs3752462 on DKD.

The association of MYH9-APOL1 is very interesting in DKD. It could provide the genetic tool to identify DM patients with increased risk of progressing to $\mathrm{DKD}$, at least in populations in whom the mutations are known to be prevalent variations. Comprehension of the biological mechanisms determining proteinuria and CKD in patients presenting these mutations can create opportunity for new therapeutic targets and measures.

In conclusion, our study suggests that MYH9 SNP rs3752462 is significantly associated with DKD in patients with $\mathrm{DM}$ and confirms the minor allele $\mathrm{C}$ as a risk factor of DKD. APOL1 rs136161 was not related to the risk of DKD in our sample, but the result can be related to our small sample size. It will be a current challenge to explore the biological mechanisms underlying this association in future research.

\section{Conflicts of Interest}

The authors declare that they have no conflicts of interest.

\section{Authors' Contributions}

Hailing Zhao and Liang Ma contributed equally to this work.

\section{Acknowledgments}

This study was supported by the National Natural Science Foundation of China (Grant nos. 81503418 and 8162010803) and a Project of International Collaboration in Science and Technology Grant, China (Grant no. 2011DFA31860).

\section{References}

[1] A. J. Collins, B. Kasiske, C. Herzog et al., "United States Renal Data System 2006 annual data report abstract," American Journal of Kidney Diseases, vol. 49, Supplement 1, pp. A6-A7, 2007.

[2] B. I. Freedman, M. Bostrom, P. Daeihagh, and D. W. Bowden, "Genetic factors in diabetic nephropathy," Clinical Journal of the American Society of Nephrology, vol. 2, no. 6, pp. 1306-1316, 2007. 
[3] R. Gupta and A. Misra, "Epidemiology of microvascular complications of diabetes in South Asians and comparison with other ethnicities," Journal of Diabetes, vol. 8, no. 4, pp. 470-482, 2016.

[4] C. Arrondel, N. Vodovar, B. Knebelmann et al., "Expression of the nonmuscle myosin heavy chain IIA in the human kidney and screening for MYH9 mutations in Epstein and Fechtner syndromes," Journal of the American Society of Nephrology, vol. 13, no. 1, pp. 65-74, 2002.

[5] S. P. Fukuda, T. S. Matsui, T. Ichikawa et al., "Cellular force assay detects altered contractility caused by a nephritisassociated mutation in nonmuscle myosin IIA," Development, Growth \& Differentiation, vol. 59, no. 5, pp. 423-433, 2017.

[6] W. H. Linda Kao, M. J. Klag, L. A. Meoni et al., "MYH9 is associated with nondiabetic end-stage renal disease in African Americans," Nature Genetics, vol. 40, no. 10, pp. 1185-1192, 2008.

[7] J. B. Kopp, M. W. Smith, G. W. Nelson et al., "MYH9 is a major-effect risk gene for focal segmental glomerulosclerosis," Nature Genetics, vol. 40, no. 10, pp. 1175-1184, 2008.

[8] J. N. Cooke, M. A. Bostrom, P. J. Hicks et al., "Polymorphisms in $\mathrm{MYH9}$ are associated with diabetic nephropathy in European Americans," Nephrology Dialysis Transplantation, vol. 27, no. 4, pp. 1505-1511, 2012.

[9] B. I. Freedman, J. B. Kopp, C. D. Langefeld et al., "The apolipoprotein L1 (APOL1) gene and nondiabetic nephropathy in African Americans," Journal of the American Society of Nephrology, vol. 21, no. 9, pp. 1422-1426, 2010.

[10] G. A. Hawkins, D. J. Friedman, L. Lu et al., "Re-sequencing of the APOL1-APOL4 and MYH9 gene regions in African Americans does not identify additional risks for CKD progression," American Journal of Nephrology, vol. 42, no. 2, pp. 99-106, 2015.

[11] S. K. Iyengar, J. R. Sedor, B. I. Freedman et al., "Genome-wide association and trans-ethnic meta-analysis for advanced diabetic kidney disease: family investigation of nephropathy and diabetes (FIND)," PLoS Genetics, vol. 11, no. 8, article e1005352, 2015.

[12] G. Dorval, O. Gribouval, V. Martinez-Barquero et al., "Clinical and genetic heterogeneity in familial steroid-sensitive nephrotic syndrome," Pediatric Nephrology, vol. 33, no. 3, pp. 473-483, 2018.

[13] S. Tzur, S. Rosset, R. Shemer et al., "Missense mutations in the APOL1 gene are highly associated with end stage kidney disease risk previously attributed to the MYH9 gene," Human Genetics, vol. 128, no. 3, pp. 345-350, 2010.

[14] B. H. Keeling and B. R. Taylor, "Keloids and non-diabetic kidney disease: similarities and the APOL1-MYH9 haplotype as a possible genetic link," Medical Hypotheses, vol. 81, no. 5, pp. 908-910, 2013.

[15] C. M. O'Seaghdha, R. S. Parekh, S. J. Hwang et al., “The MYH9/ $A P O L 1$ region and chronic kidney disease in EuropeanAmericans," Human Molecular Genetics, vol. 20, no. 12, pp. 2450-2456, 2011.

[16] M. A. Bostrom, L. Lu, J. Chou et al., "Candidate genes for nondiabetic ESRD in African Americans: a genome-wide association study using pooled DNA," Human Genetics, vol. 128, no. 2, pp. 195-204, 2010.

[17] N. Singh, N. Nainani, P. Arora, and R. C. Venuto, "CKD in MYH9-related disorders," American Journal of Kidney Diseases, vol. 54, no. 4, pp. 732-740, 2009.
[18] D. B. Johnstone, O. Ikizler, J. Zhang, and L. B. Holzman, "Background strain and the differential susceptibility of podocyte-specific deletion of Myh9 on murine models of experimental glomerulosclerosis and HIV nephropathy," PLoS One, vol. 8, no. 7, article e67839, 2013.

[19] T. Sekine, M. Konno, S. Sasaki et al., "Patients with EpsteinFechtner syndromes owing to MYH9 R702 mutations develop progressive proteinuric renal disease," Kidney International, vol. 78, no. 2, pp. 207-214, 2010.

[20] A. N. Mhatre, Y. Li, N. Bhatia, K. H. Wang, G. Atkin, and A. K. Lalwani, "Generation and characterization of mice with Myh9 deficiency," NeuroMolecular Medicine, vol. 9, no. 3, pp. 205-215, 2007.

[21] J. Crish, M. A. Conti, T. Sakai, R. S. Adelstein, and T. T. Egelhoff, "Keratin 5-Cre-driven excision of nonmuscle myosin IIA in early embryo trophectoderm leads to placenta defects and embryonic lethality," Developmental Biology, vol. 382, no. 1, pp. 136-148, 2013.

[22] D. B. Johnstone, J. Zhang, B. George et al., "Podocyte-specific deletion of $M y h 9$ encoding nonmuscle myosin heavy chain 2A predisposes mice to glomerulopathy," Molecular and Cellular Biology, vol. 31, no. 10, pp. 2162-2170, 2011.

[23] S. Cechova, F. Dong, F. Chan, M. J. Kelley, P. Ruiz, and T. H. Le, "MYH9 E1841K mutation augments proteinuria and podocyte injury and migration," Journal of the American Society of Nephrology, vol. 29, no. 1, pp. 155-167, 2018.

[24] A. A. Wasik, V. Dumont, J. Tienari et al., "Septin 7 reduces nonmuscle myosin IIA activity in the SNAP23 complex and hinders GLUT4 storage vesicle docking and fusion," Experimental Cell Research, vol. 350, no. 2, pp. 336-348, 2017.

[25] X. Fan, H. Yang, S. Kumar et al., "SLIT2/ROBO2 signaling pathway inhibits nonmuscle myosin IIA activity and destabilizes kidney podocyte adhesion," JCI Insight, vol. 1, no. 19, article e86934, 2016.

[26] G. W. Nelson, B. I. Freedman, D. W. Bowden et al., "Dense mapping of MYH9 localizes the strongest kidney disease associations to the region of introns 13 to 15," Human Molecular Genetics, vol. 19, no. 9, pp. 1805-1815, 2010.

[27] C. Ling, C. Y. Cai, B. C. Chang et al., "MYH9 gene polymorphisms may be associated with cerebrovascular blood flow in patients with type 2 diabetes," Genetics and Molecular Research, vol. 14, no. 1, pp. 1008-1016, 2015.

[28] B. Tavira, E. Coto, J. Gómez et al., “Association between a MYH9 polymorphism (rs3752462) and renal function in the Spanish RENASTUR cohort," Gene, vol. 520, no. 1, pp. 73-76, 2013. 


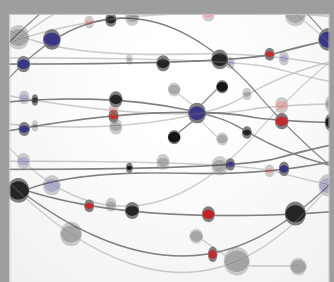

The Scientific World Journal
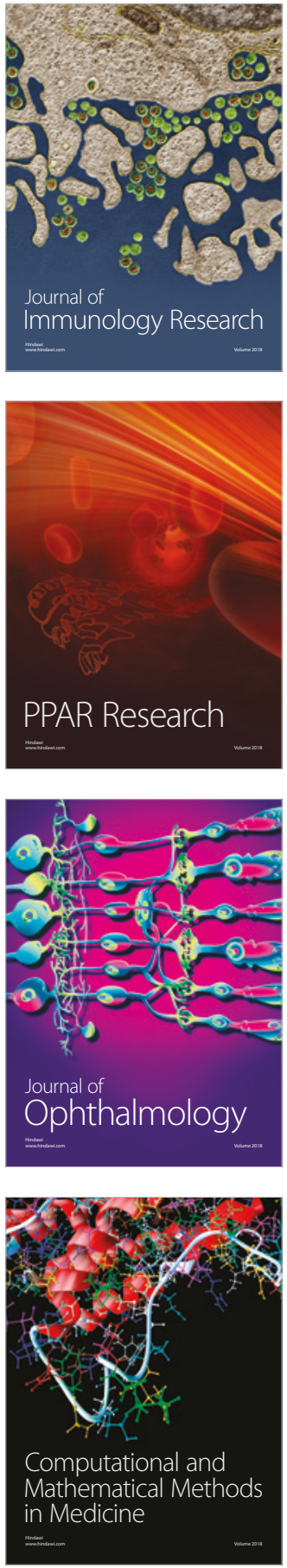

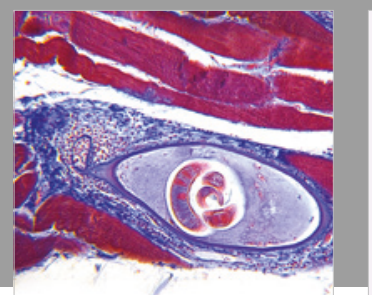

Gastroenterology Research and Practice

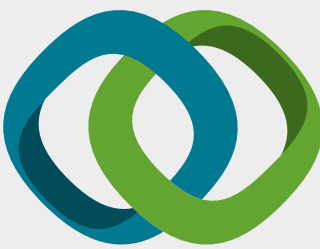

\section{Hindawi}

Submit your manuscripts at

www.hindawi.com
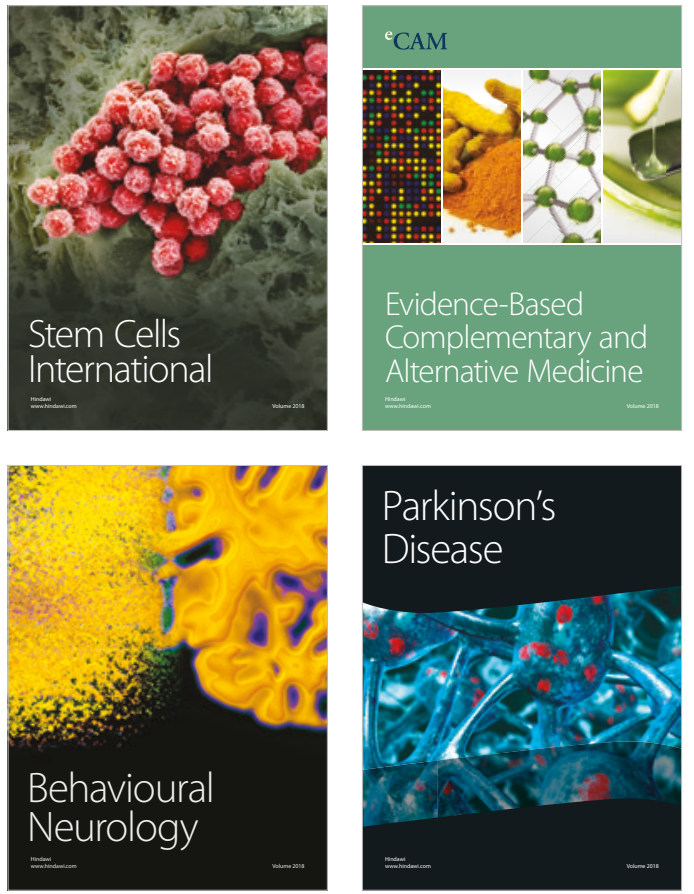

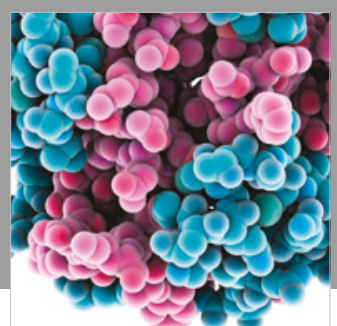

ournal of

Diabetes Research

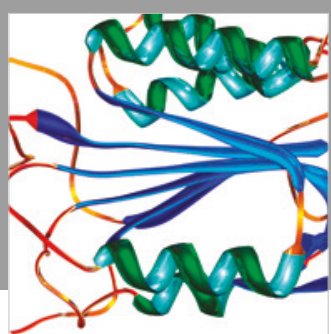

Disease Markers
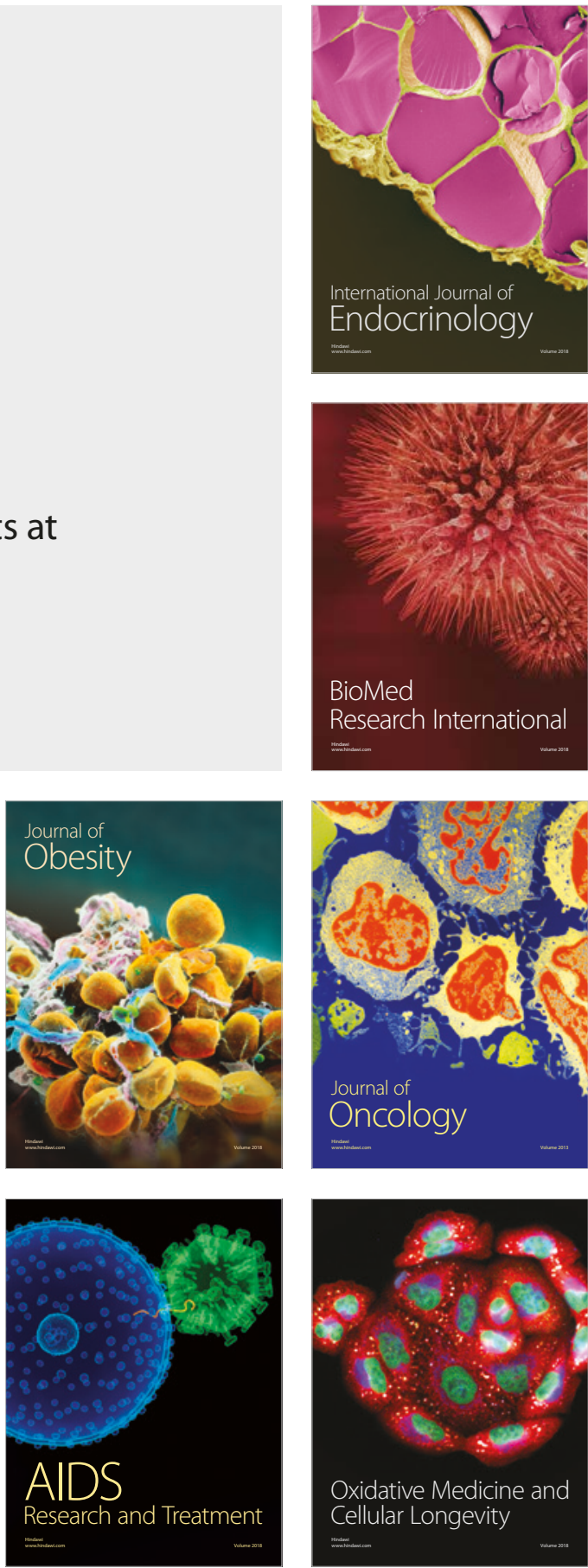